\title{
Cardiocerebral infarction with sinus node dysfunction: A management conundrum?
}

\author{
Rahul Nijhawan, Sanjeev Asotra, Prakash C Negi, Rajesh Sharma and Kunal Mahajan* \\ Department of Cardiology; Indira Gandhi Medical College and Hospital Shimla, HP, India
}

\begin{abstract}
Acute ischemic stroke (AIS) and acute myocardial infarction (AMI) are well recognized clinical entities with established management algorithms. However, concurrent cardio-cerebral infarction (CCI) is an extremely rare phenomenon with grave prognosis. Here we present a case of cardio-cerebral infarction with sinus node dysfunction and highlights the existing challenge to its management.
\end{abstract}

\section{Introduction}

Acute ischemic stroke (AIS) and acute myocardial infarction (AMI) are both life-threatening medical conditions that carry poor prognosis. In a study by Chin et al, showed that $12.7 \%$ of AIS patients suffered from AMI within 72 hours of admission [1]. Concurrent AMI and AIS pose a unique challenge for physicians as both conditions have a narrow therapeutic time-window and management of one may result in disability in the other. The dual antiplatelets and anticoagulants used in management of AMI can increase risk of hemorrhagic conversion of acute infarct. On the other hand, use of a thrombolytic in AIS increases the risk of cardiac wall rupture in the setting of AMI. Due to rarity of the condition, the management of these patients is very challenging and there is no ideal recommendation. Balanced management should be a trade-off between early rescuing the brain or the heart. Here we present a case of cardiocerebral infarction who presented to cardiology department in IGMC, Shimla.

\section{Case report}

A 75-year-old male presented to Cardiology Department with chief complaints of weakness in right upper limb, difficulty in speaking, dizziness and chest pain since last 3 days. His past medical history was significant for diabetes since last 4 years while he denied any history of hypertension or chronic smoking. Clinical examination revealed a normal built patient with vitals of BP- $120 / 70 \mathrm{mmHg}$, pulse rate (PR)$76 \mathrm{bpm}$ regular, oxygen saturation- $93 \%$ and random blood sugar of $160 \mathrm{mg} / \mathrm{dl}$. His jugular venous pressure was not raised. Cardiac examination revealed normal first and second heart sounds and no murmurs or additional heart sounds. Central Nervous system exam revealed weakness grade 3/5 in right upper limb, cerebellar signs and Babinski sign were present. Electrocardiogram was performed (Figures 1-4) and revealed T wave inversions in inferior leads II, III and avF and ST segment depression in leads V1-V6 as shown in (Figure 1). Posterior chest leads showed ST segment elevation in V7-V8. The clinical picture together with electrocardiographic findings suggested a diagnosis of acute transmural posterior wall infarction along with subendocardial ischemia of inferior, anterior and lateral walls. 2D-echocardiograpy was done and revealed akinetic posterior wall with moderate left ventricular systolic dysfunction and no left ventricular (LV) clot or mechanical complication.
A computed tomography of the brain (Figure 5) was performed and revealed hypodensity in left temporo-parieto-occipital lobe involving both grey and white matter. Magnetic resonance imaging (MRI) Brain (Figure 6) revealed subacute infarct in left frontal and left occipital region. Lab investigations revealed evidence of myocardial damage with elevation of cardiac enzymes and troponin levels. Serum myoglobin levels were $>500 \mathrm{ng} / \mathrm{ml}$, creatinine kinase (CK-MB) $46.4 \mathrm{ng} / \mathrm{ml}$ and troponin I $15.5 \mathrm{ng} / \mathrm{ml}$. Coronary angiography revealed triple vessel disease, with $95 \%$ stenosis in mid right coronary artery (RCA), $60 \%$ stenosis in left circumflex (LCX) and 50\% stenosis in mid-Left anterior descending (LAD) (Figures 7-9) Since the patient had presented on the third day of symptoms and was having no chest pain at that time, we decided to manage conservatively with dual antiplatelet, statins, beta blockers, and angiotensin converting enzyme (ACE) inhibitors. On the third day of hospital stay, patient had multiple episodes of lightheadedness and resting syncope. No hypotension was documented. However, electrocardiogram (Figure 10) showed multiple episodes of atrial fibrillation followed by sinus pauses, which required temporary pacing. Patient denied any chest pain. We believed the sinus node dysfunction to be non-ischemic, and the use of beta blockers could have unmasked the underlying sinus node dysfunction, so we stopped beta blockers. Patient's sinus rhythm returned, and temporary pacing was removed on 5th day of hospital stay. Although his limb weakness persisted, but since the patient was chest pain free and was in sinus rhythm, he was discharged on day 8 . Patient's attendants were not willing for a further electrophysiological work-up for sinus node evaluation. However, they were advised that the patient might require a permanent pacemaker implantation in near future considering the underlying sick sinus syndrome.

${ }^{*}$ Correspondence to: Kunal Mahajan, Assistant Professor, Department of cardiology; Indira Gandhi Medical College and Hospital Shimla, HP 171001, India, Tel: +91-8628864820, E-mail: kunalmahajan442@gmail.com

Key words: cardiocerebral infarction, sinus node, stroke, myocardial infarction

Received: April 15, 2020; Accepted: April 27, 2020; Published: May 06, 2020 


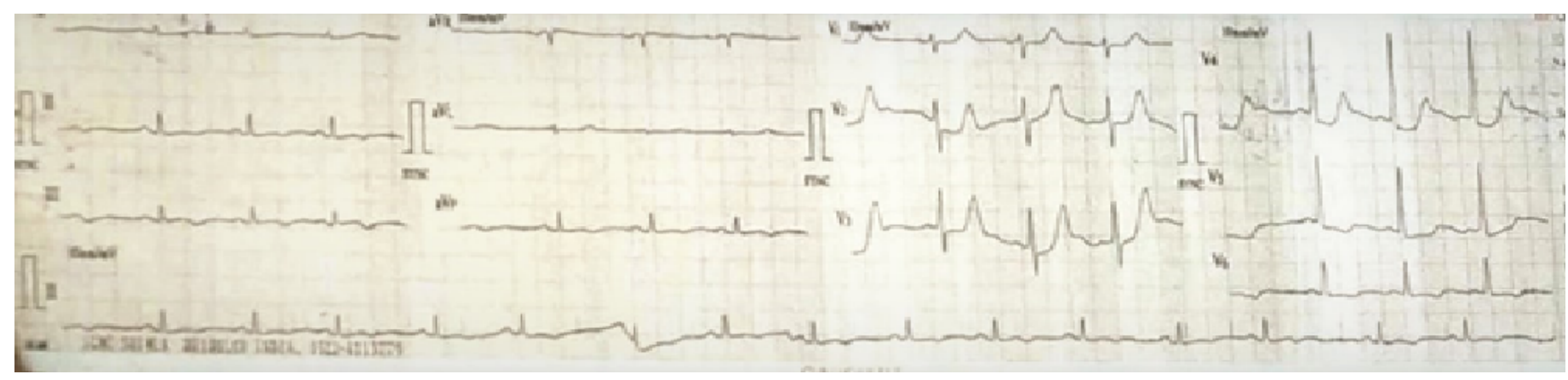

Figure 1. ECG at presentation showing $\mathrm{t}$ wave inversions in inferior leads and ST depression in anterior leads

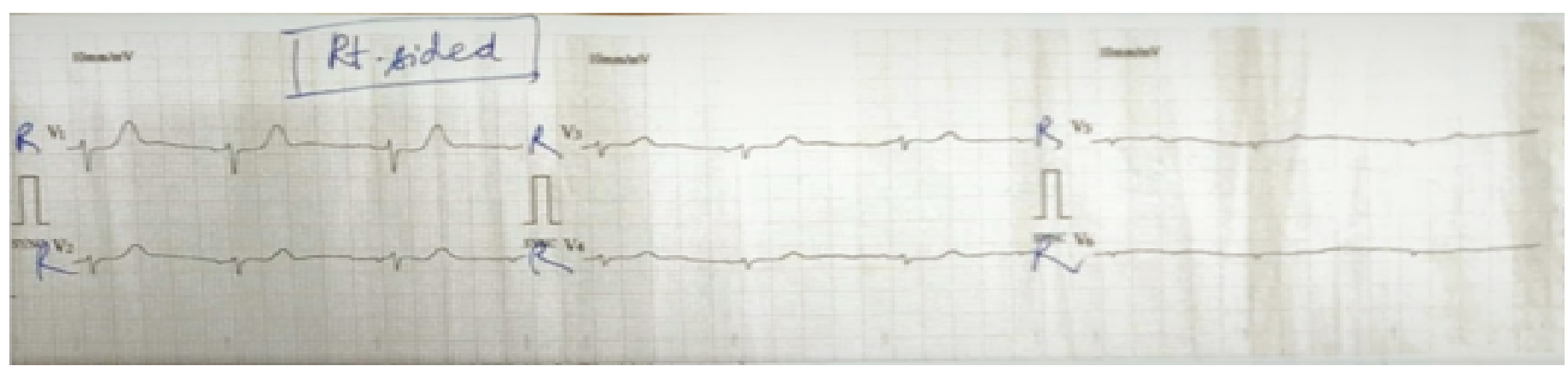

Figure 2. ECG showing normal ST segment in right sided chest lead v4R

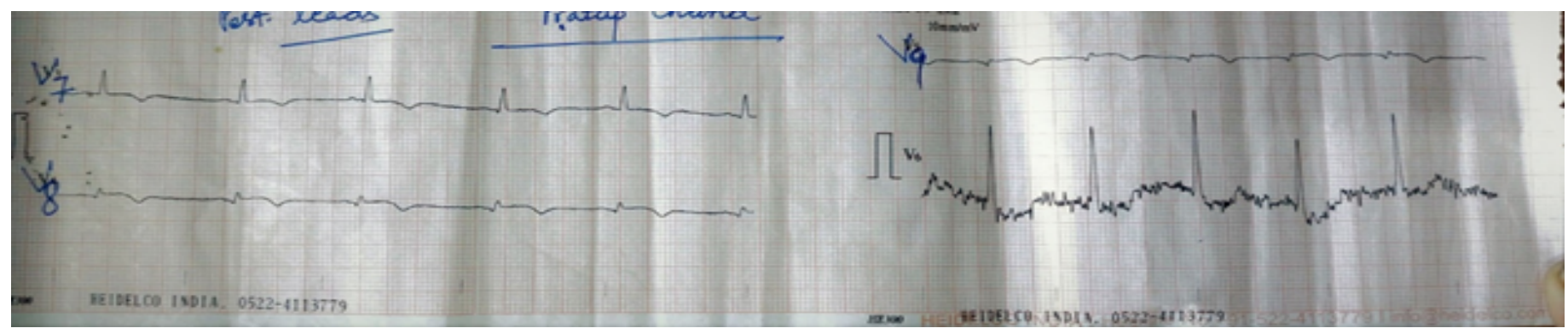

Figure 3. ECG showing ST elevation in posterior leads v8,v9

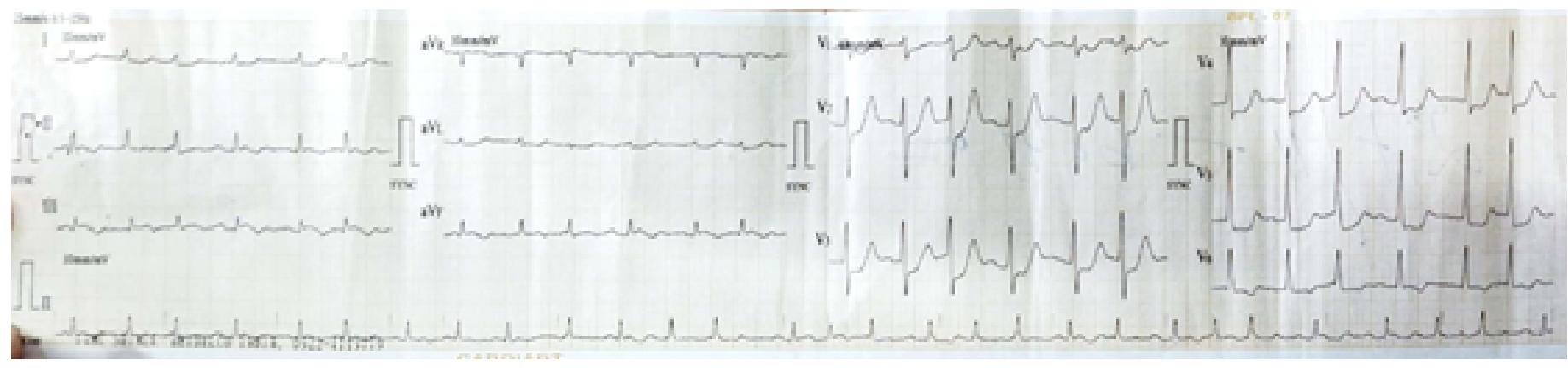

Figure 4. ECG showing atrial fibrillation on day 3 of admission 


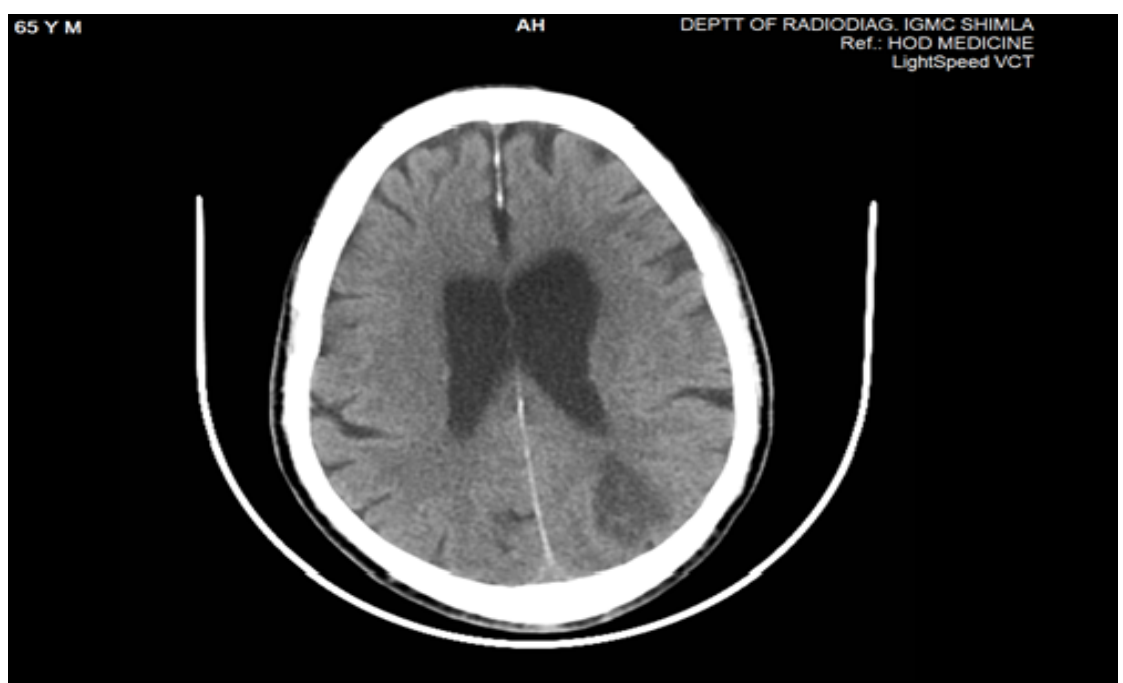

Figure 5. NCCT Head showing hypodensity involving left parieto-occipital region

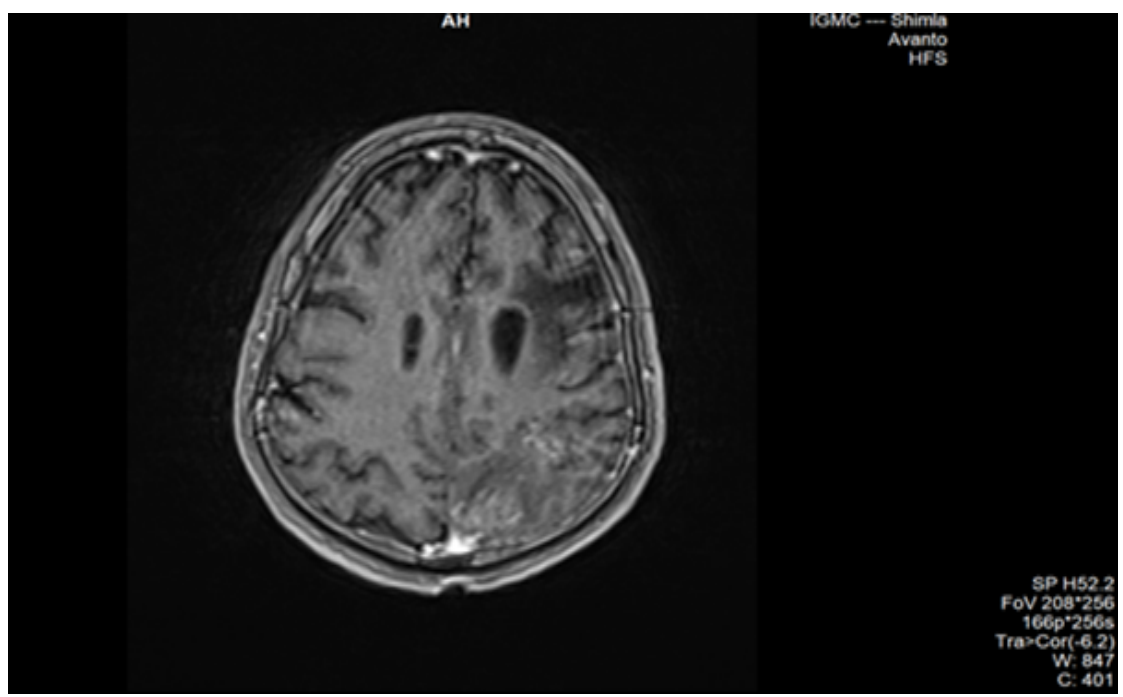

Figure 6. MRI showing subacute infarct involving left frontal and left occipital region

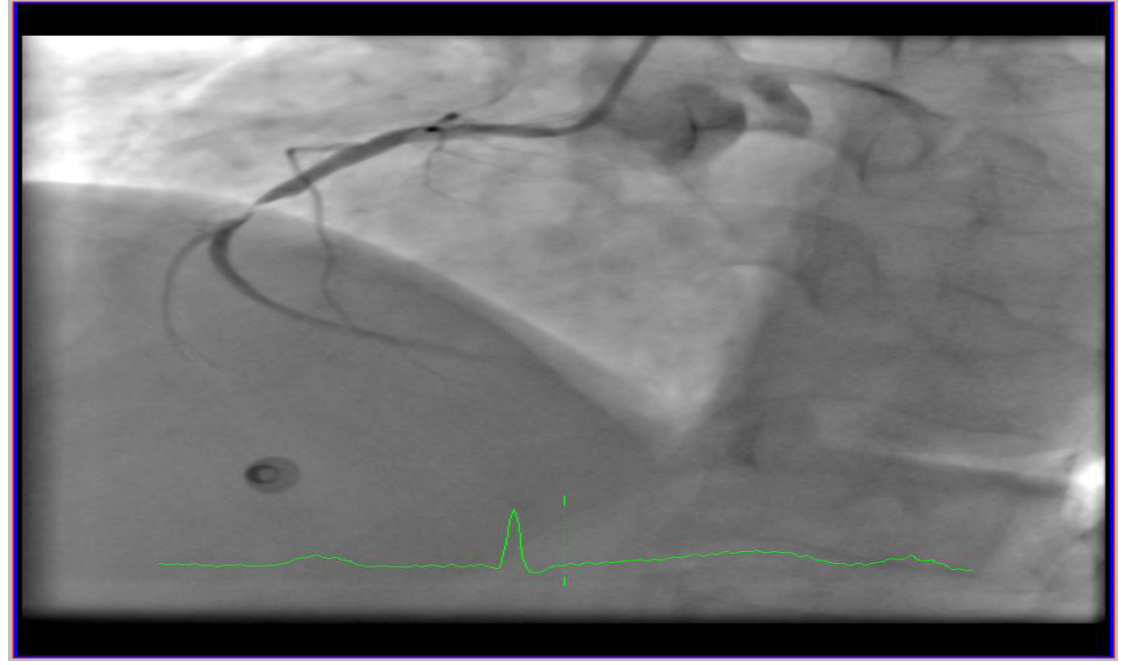

Figure 7. Coronary angiography showing mid RCA $95 \%$ stenosis 


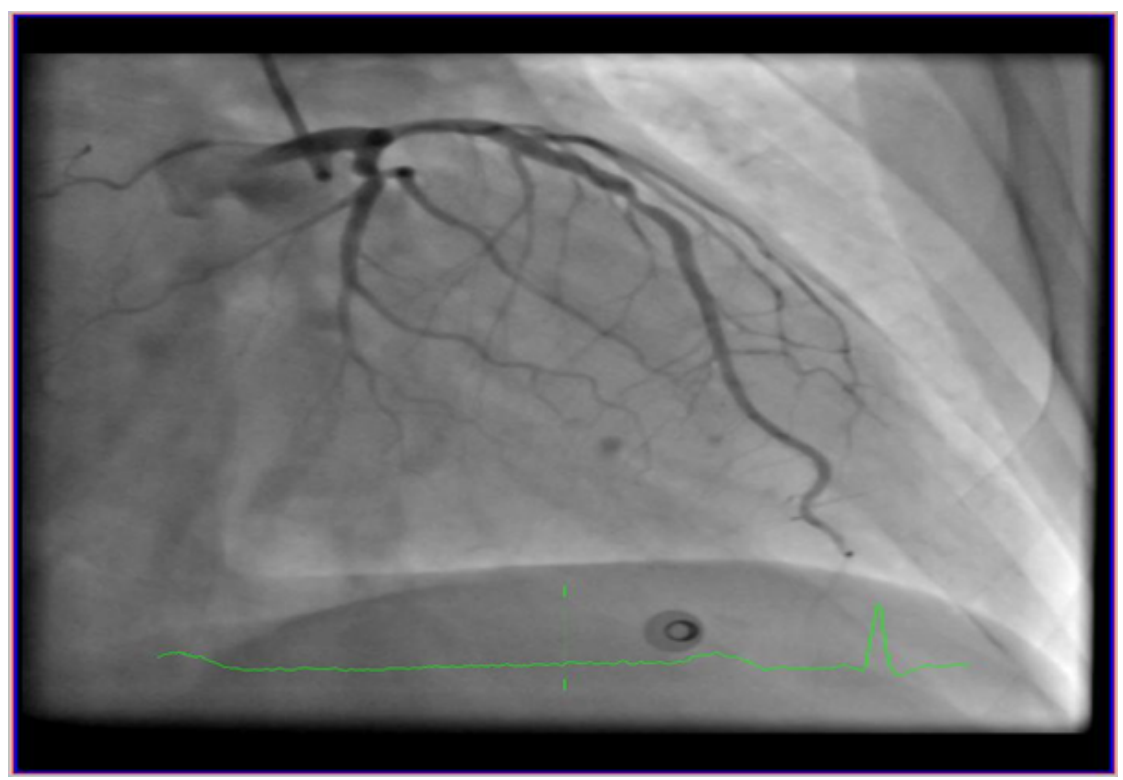

Figure 8. Coronary angiography showing mid LAD 50\% stenosis

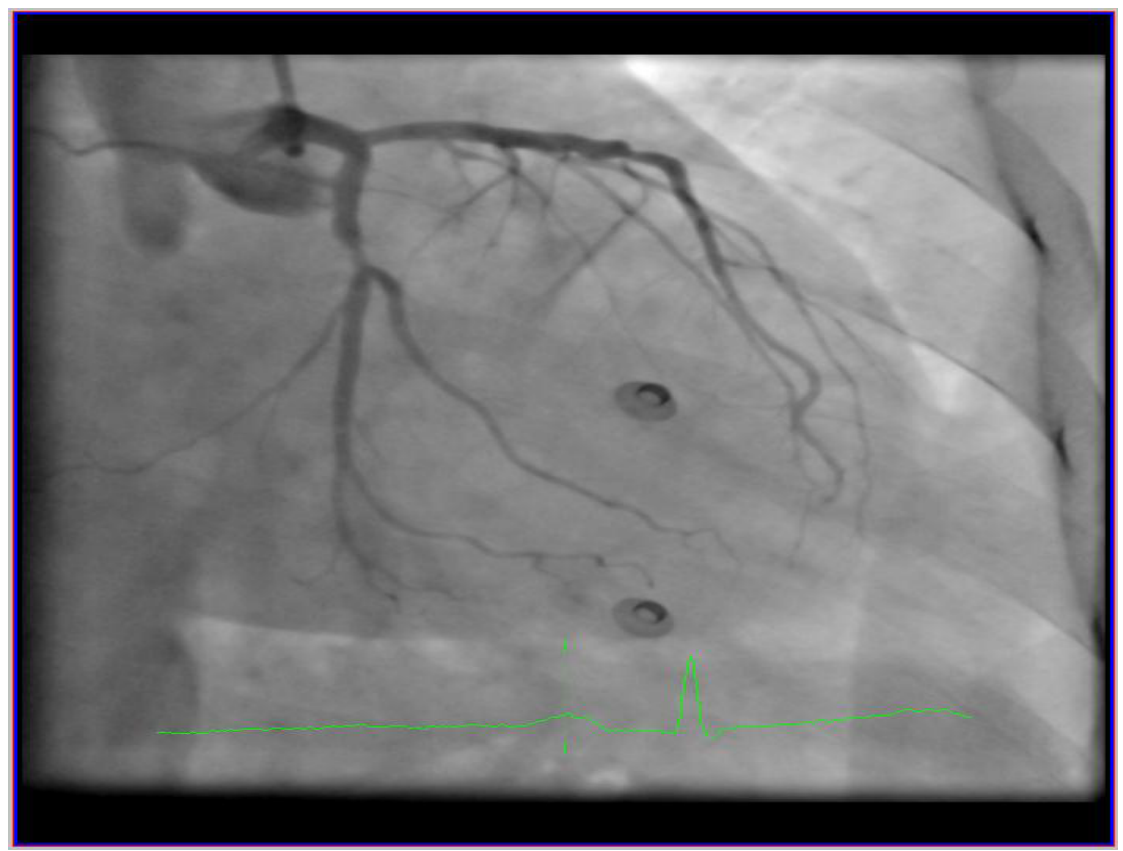

Figure 9. Coronary Angiography showing mid LCX 60\% stenosis

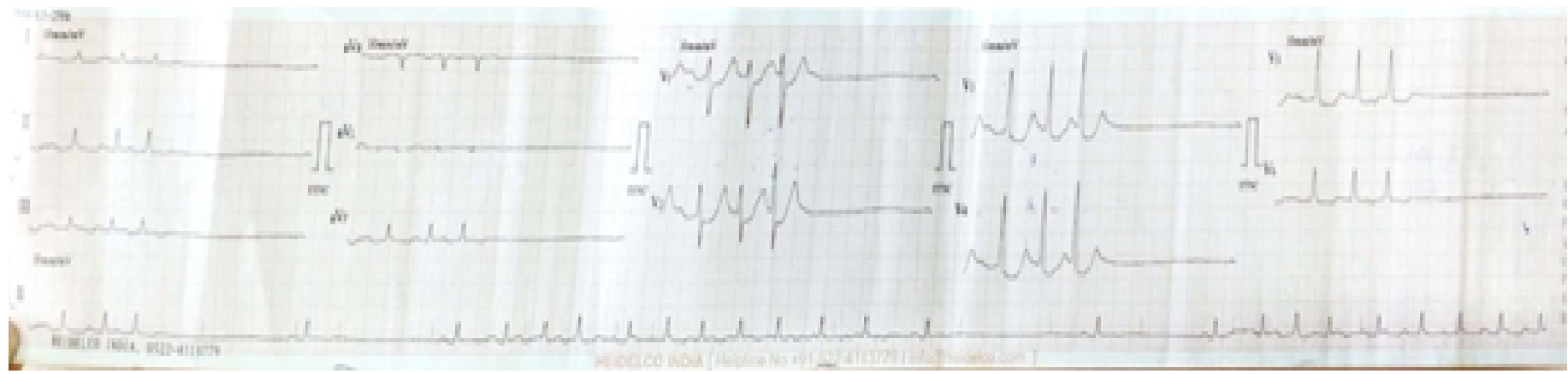

Figure 10. ECG showing atrial fibrillation followed by sinus pause, suggestive of sinus node dysfunction in the form of tachy-brady syndrome 


\section{Discussion}

Concomitant acute myocardial infarction and ischemic cerebrovascular strokes has been rarely reported in literature, with an incidence of $0.009 \%[1,2]$. According to the Austrian stroke unit registry, only $1 \%$ of patients with ischemic stroke or TIA suffered from MI [3]. While $0.9 \%$ patients of MI patients develop ischemic stroke during hospital stay [4]. Kijpaisalratana et al in their review of 8 cases of simultaneous cardiocerebral infarction found that all the ischemic strokes occurred in large vessel territories and majority of them had ST elevation inferior wall MI [5]. Our patient also had posteroinferior wall MI and ischemic stroke occurred in left middle cerebral and posterior cerebral artery territories. Various mechanisms have been proposed to explain this phenomenon. It has been postulated that systemic hypotension due to MI can precipitate cerebral infarction, second, thromboembolism from the heart to cerebral vessels. Thirdly, a massive cerebral infarction accounting for ECG changes suggestive of MI. Fourth, cerebral infarction precipitating vasomotor derangement and resulting in a MI. Other plausible explanations include presence of patent foramen ovale (PFO) causing paradoxical embolism in right ventricular myocardial infarction (RVMI) and dissection of ascending aorta extending to right coronary ostia, then to involve vertebral artery and further extension to involve basilar artery [1]. Most likely mechanism in our patient could be thromboembolism from heart to cerebral vessels. No hypotension was documented during hospital stay but systemic hypotension due to MI precipitating ischemic stroke cannot be ruled out, as patient presented to us on day 3 of symptomology. However, we did not find any evidence supporting hypotension in history nor in lab investigations. Chin et al in their prospective study of acute cerebrovascular accident patients found $12.7 \%$ incidence of associated MI. The mortality rate for the combined acute MI and cerebral infarction was 53\% at 6 weeks, $64 \%$ in one year [1]. This case of cardiocerebral infarction is a rare and complicated case that carries a poor prognosis. No guidelines have mentioned the management of this medical emergency. Kijpaisalratana et al have proposed a management algorithm for its management, which is guided by hemodynamic status of the patient. Emergency PCI is advocated in patients with hemodynamic instability followed by endovascular treatment for ischemic stroke in case of large vessel occlusion. In stable patients, intravenous recombinant tissue plasminogen activator should be administered initially at a dose of $0.9 \mathrm{mg} / \mathrm{kg}$ and endovascular therapy is performed in cases of large vessel occlusion [5]. Further studies are needed to determine the role of autonomic dysfunction, $\mathrm{PFO}$ in the pathogenesis of cardio-cerebral infarction.

\section{Authors Contribution}

All the authors were actively involved in the management of the patient and preparation of this manuscript. Manuscript has been read and approved by all the authors, and the requirements for authorship have been met, and each author states that the manuscript represents honest work.

\section{References}

1. Chin PL, Kaminski J, Rout M (1977) Myocardial infarction coincident with cerebrovascular accidents in the elderly. Age Ageing 6: 29-37. [Crossref]

2. Yeo LL, Andersson T, Yee KW, Tan BY, Paliwal P, et al. (2017) Synchronous cardiocerebral infarction in the era of endovascular therapy: which to treat first? $J$ Thromb Thrombolysis 44: 104-111. [Crossref]

3. Gattringer T, Niederkorn K, Seyfang L, Seifert-Held T, Simmet N, et al. (2014) Myocardial infarction as a complication in acute stroke: results from the Austrian Stroke Unit Registry. Cerebrovasc Dis 37: 147-152. [Crossref]

4. Saczynski JS, Spencer FA, Gore JM, Gurwitz JH, Yarzebski J, et al. (2008) Twentyyear trends in the incidence of stroke complicating acute myocardial infarction: Worcester heart attack study. Arch Intern Med 168: 2104-2110. [Crossref]

5. Kijpaisalratana N, Chutinet A, Suwanwela NC (2017). Hyperacute simultaneous cardiocerebral infarction: rescuing the brain or the heart first? Front Neurol; 8: 664. [Crossref]

Copyright: (C2020 Nijhawan R. This is an open-access article distributed under the terms of the Creative Commons Attribution License, which permits unrestricted use, distribution, and reproduction in any medium, provided the original author and source are credited. 